\title{
FECUNDIDAD EN LA ADOLESCENCIA: UNA BREVE REVISIÓN DE LITERATURA
}

\section{RAYMER DíAZ-HERnÁNDEZ}

Recibido: marzo, 2019• Aceptado: agosto, 2019

Cómo citar: Díaz-Hernández, R. (2019). Fecundidad en la adolescencia: una breve revisión de literatura. Ciencia, Economía y Negocios, 3(1), 37-47. Doi: https://doi.org/ 10.22206/ceyn.2019.v3i1.pp37-47

\section{Resumen}

El contenido de este trabajo resume la literatura existente, tanto en la ciencia económica como en otras disciplinas de las ciencias sociales, relacionada con la fecundidad en la adolescencia en general y para República Dominicana, especificamente. En sintesis, se establecen dos caracteristicas generales sobre los trabajos de investigación: 1) la fecundidad adolescente es un fenómeno que afecta particularmente a las adolescentes de estratos socioeconómicos más bajos; 2) dados los resultados mixtos sobre las distintas politicas públicas que buscan reducir la fecundidad adolescente, no existe consenso sobre cuál de estas es la mejor. Se concluye con las investigaciones más recientes, que sugieren enfocar las políticas públicas de reducción de la fertilidad en la adolescencia hacia la promoción de estilos de vida distintos a la maternidad, por ejemplo, en la obtención de mayores niveles educativos. También se sugieren posibles temas de estudio relacionados con la fecundidad en la adolescencia en República Dominicana.

Palabras clave: fecundidad adolescente; políticas públicas; educación sexual.

Códigos JEL: J13, J18.

aDíaz Hernández, Raymer: Banco Central de la República Dominicana: Av. Pedro Henríquez Ureña esq. Av. Leopoldo Navarro, Santo Domingo de Guzmán, D.N., República Dominicana. T: 809-221-9111, correo-e: raymerd@gmail.com).

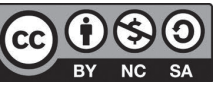




\title{
Adolescent fERTILTy: A BRIEF LITERATURE REVIEW
}

\author{
RAYMer DíAz-Hernández
}

Received: March, 2019• Approved: August, 2019

\begin{abstract}
This paper overviews the existing literature, both in economics as in other related social sciences, regarding adolescent fertility in general and in the Dominican Republic. In short, two main conclusions emerge from reviewing the research: 1) adolescent fertility is a phenomenon that affects mostly teenage girls from low socioeconomic status; 2) given the mixed results on the different public policies that seek to reduce adolescent fertility, there is no consensus regarding which existing public policy works best towards this goal. This review concludes with the most recent research, which suggests that public policy should focus on promoting lifestyles different from motherhood on teenage girls, such as getting higher education. I also propose possible study topics regarding adolescent fertility in the Dominican Republic.
\end{abstract}

Keywords: teen fertility; public policy; sexual education.

JEL Codes: J13, J18. 


\section{Introducción}

La fecundidad adolescente en República Dominicana ha cobrado importancia entre el sector público y organismos de la sociedad civil. Al respecto, la posición del país en relación con la de otros países de la región ha sido una de las principales razones del reciente interés sobre el tema. De acuerdo con las estimaciones más recientes del World Population Prospects 2017, República Dominicana poseía la tasa de fecundidad de madres adolescentes más elevada de toda América Latina y el Caribe, con 96.1 por cada mil nacimientos. Esta tasa contrasta con la de países con menor ingreso per cápita, como Bolivia (69.0 por mil nacimientos), Ecuador (74.6 por mil nacimientos) y Haití (38.1 por mil nacimientos).

Esta situación con las altas tasas de fecundidad adolescente supone una segunda fuente de preocupación, con consecuencias que se reflejan en menores niveles de escolaridad luego de las adolescentes ser madres y mayores riesgos de salud para las jóvenes durante el parto (Programa de las Naciones Unidas para el Desarrollo de República Dominicana, 2017). La maternidad adolescente implica costos importantes para el sistema de salud. Se estima el gasto total directo para los servicios de atención al embarazo en adolescentes en general en $\mathrm{RD} \$ 2,103.3$ millones, 1.7 veces mayor al presupuesto de servicios de salud colectiva con recursos internos, lo que representa el $5 \%$ del gasto público total en salud presupuestado para el país (Programa de Estudios Sociodemográficos, 2013).

El presente documento consiste en una resumida revisión de la literatura existente acerca de las causas del embarazo y la maternidad en la adolescencia. Es necesario aclarar, en primer lugar, que en esta ocasión, no se pretende abarcar todo lo escrito sobre el tema, en cambio se optó por enfocar este documento en estudios seleccionados que permitan despertar el interés y ofrece al lector una rápida comprensión sobre los aspectos más relevantes de la fecundidad en adolescentes en el país.

En segundo lugar, aunque la mayoría de las investigaciones que se presentarán en este análisis provienen de la ciencia económica, también se citan otros trabajos asociados a otras ramas de las ciencias sociales, principalmente de la sociología. Existen diferencias importantes respecto a la metodología de investigación desde ambas ramas, y cada una ofrece sus ventajas y desventajas. La economía, por ejemplo, suele auxiliarse 
de los resultados de encuestas como las Encuestas Demográficas de Salud (ENDESA), que permiten hacer generalizaciones respecto a la población. A su vez, una limitante de la que adolecen las inferencias a partir de encuestas es que no permiten explorar hipótesis más allá de las preguntas encontradas en las encuestas. Por otra parte, la sociología, a través de herramientas como las entrevistas a profundidad, permiten investigar con mayor detalle sobre lo que piensan las adolescentes tanto previo como posterior al embarazo y/o parto; una desventaja de esta herramienta, no obstante, es que su profundidad de análisis no permite realizar generalizaciones respecto al resto de la población. ${ }^{a}$

\section{¿Qué produce la fecundidad en la adolescencia?}

Desde la sociología, el embarazo y la fecundidad en la adolescencia se aborda como el resultado de la interrelación entre un cúmulo de factores directamente vinculados al entorno familiar en el que se desarrollan las niñas y adolescentes, tales como la presión que se ejerce desde su círculo más cercano y los niveles de ingreso y educación de la familia (Agüero-Reyes, 2005). En este aspecto, Álvarez-Nieto, Pastor-Moreno, Linares-Abada, Serrano-Martos y Rodríguez-Olalla, (2012) afirman que si bien la fecundidad puede darse en el marco de relaciones afectivas, las adolescentes racionalizan su condición de embarazo o maternidad una vez ocurre; a su vez, los autores atribuyen como causa principal del embarazo o fecundidad tanto a la falta de información como al desuso de métodos anticonceptivos.

Por otra parte, los economistas Melissa Kearny y Phillip Levine (2014) apuntan a la fecundidad en la adolescencia como un síntoma de las condiciones de pobreza en la que vive la población, en vez de ser una causante de ella. En el contexto estadounidense, estos autores estiman que las adolescentes de bajo estatus socioeconómico de Estados Unidos tienen mayor probabilidad de procrear a edad temprana cuando viven en estados de mayor desigualdad; las diferencias de ingreso entre estados permiten explicar la disparidad en las tasas de fecundidad interestatal. Las implicaciones de estos resultados son importantes, ya que llevan a pensar que

aLas metodologías de PNUD-RD (2017) y PESD (2013) representan un importante avance en este sentido, ya que combina tanto el análisis cuantitativo como el cualitativo. 
la mejora en las oportunidades económicas y la reducción de la pobreza serían los principales motores de la reducción de la fecundidad en la adolescencia. $^{\mathrm{b}}$

Otro estudio que también asocia la maternidad en la adolescencia al entorno socioeconómico de las madres es el de Odimegwu y Mkwananzi (2016), quienes indican que los distintos niveles de desigualdad a nivel comunitario conducen a disparidades en la salud reproductiva entre mujeres adolescentes de África subsahariana, al generar desventajas sociales en las adolescentes. Factores como la disrupción familiar y la pobreza y desempleo en las mujeres a nivel de las comunidades inciden en mayores tasas de fecundidad para la región.

\section{¿Puede la educación sexual prevenir los embarazos en adolescentes?}

Dado que la maternidad en la adolescencia se produce y se reproduce en contextos de pobreza y desigualdad, que conlleva riesgos que ponen en peligro las vidas tanto de la madre como del infante y que representa un costo adicional al sistema de salud, es de interés para quienes hacen políticas públicas el reducir la incidencia de la maternidad adolescente en la sociedad. Sin embargo, las propuestas de programas que apunten a esta vía son fuente de discusión cuando se proponen. Algunas de las soluciones posibles para reducir los embarazos a edad temprana son la educación sexual en las escuelas y una mayor disponibilidad y acceso a métodos anticonceptivos. Sin embargo, ambas medidas suelen ser rechazadas por algunos sectores de la sociedad, bajo el argumento de que resultarían en promover una mayor actividad sexual entre adolescentes (Sabia, 2006).

Existe evidencia para apoyar este argumento. Oettinger (1999) analiza teóricamente los efectos de diversas políticas a partir de un análisis costo-beneficio para las adolescentes. De acuerdo con el investigador, políticas relacionadas a enseñar o desmitificar sobre el uso de anticonceptivos podrían incrementar el embarazo y la fecundidad en la adolescencia, mediante un mayor incremento en la actividad sexual.c Similarmente, Paton (2004) encuentra que el incremento en el número de sesiones clínicas

\footnotetext{
bKearny y Levine (2012).

'También ver Arcidiacono, Kwhaja, y Ouyang (2005).
} 
sobre planificación familiar juvenil incrementó el reporte de diagnósticos de infecciones de transmisión sexual en $1.5 \%$ en Inglaterra.

Sin embargo, otros trabajos aportan evidencia favorable para la educación sexual como forma de combatir los embarazos en edades temprana. Bailey (2012), por ejemplo, concluye que la introducción de programas de planificación familiar se asocia a reducciones en la fecundidad, impulsadas por el retraso en la decisión de procrear y de llevar a término el embarazo. Recientemente, Kelly, Lindo y Packham, (2019) encuentran que la "Iniciativa de Planificación Familiar del Estado de Colorado" redujo los partos en $20 \%$ entre adolescentes de 15 a 19 ańos que residían a siete millas (11.2 kilómetros) o menos de las clínicas de planificación. Por igual, Bass (2018) encuentra que: a) la educación sexual basada en la abstinencia incrementa la probabilidad de tener actividad sexual reciente y de tener múltiples compañeros sexuales; b) la educación sexual exhaustiva incrementa el uso de preservativos durante la relación sexual más reciente; c) la educación sexual no específica incrementa de forma marginal la probabilidad de tener relaciones sexuales de forma reciente.

Por otro lado, existen estudios que no encuentran efectos de la educación sexual en el comportamiento sexual de adolescentes. En este sentido, Sabia (2006) usa información del "Estudio Longitudinal Nacional de Salud Adolescente de Estados Unidos entre 1994 y 2001” para investigar si la educación sexual afecta el comportamiento y salud sexual entre adolescentes. Sus resultados indican que, si bien los adolescentes expuestos a programas sí comenzaron a tener relaciones sexuales, no fue debido al programa de educación sexual. Por tal razón, el autor concluye que los programas de educación sexual en la escuela que experimentaron los individuos dentro de la muestra no tuvieron efecto alguno en el comportamiento entre adolescentes.

En conjunto, los resultados presentados en esta revisión de literatura no aportan una evidencia contundente sobre el efecto de la educación sexual en la reducción de la fecundidad en la adolescencia. Esto no quiere decir que implementar educación sexual y aumentar el acceso a anticonceptivos no tendrían ningún efecto, ya que ambas medidas podrían proveer conocimiento importante a la población adolescente sobre estos temas. Lo que sí se plantea, en cambio, es la necesidad de evaluar los programas para poder conocer si lograron su objetivo o no, y la razón de su éxito o su fracaso. 


\section{¿Qué dice la literatura sobre República Dominicana?}

Como se destacó anteriormente, se estima que República Dominicana posee la tasa más alta de fecundidad entre mujeres adolescentes en la región. Es importante destacar que, igual a lo señalado por otros estudios, la maternidad en la adolescencia no es un fenómeno aleatorio en el país, siendo más prevalente en los hogares de estratos socioeconómicos más bajos. De acuerdo con ENDESA 2013, 8.7 \% de las mujeres dominicanas entre 15 y 19 ańos del quintil de riqueza más alto son madres o estuvieron embarazadas alguna vez; en contraste, este porcentaje aumenta a $35.1 \%$ para el quintil más bajo de riqueza. ${ }^{\mathrm{d}}$

Este hecho ha motivado a la investigación de sus posibles causas en el país. Nicolás y Mejía (2015) y Díaz y Olivo (2012) encuentran que la edad de la joven, el tener una relación de pareja y el uso de anticonceptivos son de las variables que tienen mayor incidencia en la probabilidad de tener un hijo durante la adolescencia. Carrasco (2012), por otra parte, analiza la Encuesta de Evaluación de Protección Social 2010 y encuentra que los hogares de jefatura femenina están asociados a una mayor probabilidad de contener madres adolescentes; en este sentido, es importante destacar que la fuente de información de Carrasco son aquellos hogares de bajo ingreso.

Por otra parte, los estudios sociológicos agregan que, para el contexto dominicano, los factores culturales se combinan con los ámbitos de pobreza para generar madres adolescentes. Por ejemplo, el Observatorio de Políticas Sociales y Desarrollo (2017) indica que, dado los contextos de pobreza y exclusión, el embarazo y/o la maternidad se convierten en proyectos de vida para las adolescentes. Por tal razón, Pérez-Then, Miric y Vargas (2011) concluyen que la maternidad en la adolescencia no debe verse como un accidente; en el caso de aquellas adolescentes con parejas estables, podría tratarse de un proyecto de vida impulsado por el entorno social, si bien adelantado. También argumentan que si ese proyecto de vida incluye la expectativa de la responsabilidad económica de la pareja, las adolescentes buscarán una pareja mayor de edad, lo que crea a su vez un desbalance de poder en la relación.

${ }^{\mathrm{d}}$ CESDEM (2014). 


\section{Conclusiones}

Como puede apreciarse en esta breve revisión, la literatura relacionada a las causas de la fecundidad en la adolescencia es extensa, así como las discusiones de política que conllevan. Sin embargo, se pueden extraer algunas líneas generales.

En primer lugar, la fecundidad en la adolescencia no es un fenómeno al que están aleatoriamente expuestas las jóvenes de todos los estratos sociales. Existen claras evidencias de que es un hecho que afecta en mayor cuantía los entornos de pobreza y exclusión dentro del cual la maternidad se percibe como un proyecto de vida para las adolescentes. En segundo lugar, no existe una solución única para reducir la fecundidad en la adolescencia, dado que la evidencia publicada encuentra resultados muy dispares entre sí.

Estos resultados generales hacen pensar en vías alternativas para la implementación de políticas públicas. Los programas de reducción de maternidad en la adolescencia, además de enfocarse en los estratos sociales de menores recursos, podrían orientarse en ofrecer proyectos de vida distintos a la maternidad, dirigiéndose por ejemplo en la obtención de mayores niveles educativos. Esta idea no es nueva, ya que trabajos empíricos han explorado cómo los cambios en los paradigmas culturales afectan la salud sexual y reproductiva. Por ejemplo, La Ferrara, Chong y Duryea (2008) encuentran que la presencia de canales de telenovelas en ciertas comunidades está relacionada a reducciones en la fecundidad, y a mayores tasas de escolaridad entre mujeres; esto se debe al contenido de muchas telenovelas que presentaban a mujeres en labores profesionales y ejecutivas, distintas al trabajo doméstico. Por otra parte, Jensen y Oster (2009) asocian la introducción de televisores en la India con menor aceptación de la violencia doméstica, mayor preferencia hacia la tenencia de hijas e incrementos en la escolaridad de los niños en general.

Estos trabajos apuntan a posibles mejoras en políticas de prevención de la maternidad en la adolescencia en República Dominicana, las cuales han tomado protagonismo en la última década. El Gabinete de Coordinación de Políticas Sociales ha dado prioridad a la prevención de embarazos y maternidad a partir de varias medidas, reflejándose en el apoyo a 
la elaboración de un Plan Nacional para la Reducción de Embarazos en Adolescentes (PREA-RD) donde se establecen tanto las directrices como los indicadores y líneas base. Respecto a este estudio, y sobre los planteamientos establecidos anteriormente, se reitera la necesidad de monitorear y evaluar este programa en todas las fases de su implementación.

Por último, quedan otros temas por explorar respecto al a la fecundidad en edades tempranas, tales como las evaluaciones de políticas ya implementadas que buscan reducir los embarazos tempranos, o temas relacionados como el matrimonio y fecundidad infantil (entendidos como aquellos matrimonios o embarazos que involucran a niños y niñas menores de 15 años). El estudio de estos fenómenos resulta de importancia, ya que ayudarían a tener una mejor visión sobre cómo tratar la fecundidad en la adolescencia.

\section{Referencias}

Agüero-Reyes, Z. (2005). Teen Pregnancy in Mexican-American Girls. Texas Tech University Libraries.

Álvarez-Nieto, C., Pastor-Moreno, G., Linares-Abada, M., Serrano-Martos, J., \& Rodríguez-Olalla, L. (nov/dic de 2012). Motivaciones para el embarazo adolescente. Gaceta Sanitaria, 26(6), 497-503.

Arcidiacono, P., Kwhaja, A., \& Ouyang, L. (2012). Habit Persistence and Teen Sex: Could Increased Access to Contraception Have Unintended Consequences for Teen Pregnancies? Journal of Business \& Economic Statistics, 30(2), 312-325.

Bailey, M. J. (2012). Reexamining the Impact of Family Planning Programs on U.S. Fertility: Evidence from the War on Poverty and the Early Years of Title X. American Economic Journal: Applied Economics, American Economic Association, 4(2), 62-97.

Bass, B. (25 de agosto de 2018). Let's Talk About Sex Education: The Effect of State-Mandated School-Based Sex Education on Teenage Sexual Behaviors and Health. Obtenido de brittanyebass.com/research

Carrasco, H. (2012). El Embarazo Adolescente en Hogares Pobres de la República Dominicana: Evidencia a Partir de la EEPS 2010. Obtenido de https://issuu.com/horacerord/docs/embarazo_adolescente_y_hogares_pobr 
Centro de Estudios Sociales y Demográficos (CESDEM). (2014). Encuesta Demográfica y de Salud República Dominicana 2013. Santo Domingo: CESDEM y ICF International.

Díaz, R., \& Olivo, K. (2012). Determinantes de la fecundidad en las adolescentes en República Dominicana: Evidencia a partir de Encuesta Demográfica y de Salud 2007. En B. C. Dominicana, Nueva Literatura Económica DominicanaL Premios de la Biblioteca "Juan Pablo Duarte” 2011 (págs. 131-198). Santo Domingo: Banco Central de la República Dominicana.

Jensen, R., \& Oster, E. (2009). The Power of TV: Cable Television and Women's Status in India. NBER Working Papers 13305.

Kearny, M. S., \& Levine, P. B. (2012). Why is the Teen Birth Rate in the United States So High and Why Does It Matter? Journal of Economic Perspectives, 26(2), 141-166.

Kearny, M. S., \& Levine, P. B. (2014). Income Inequality and Early Nonmarital Childbearing. Journal of Human Resources, 49(1), 1-31.

Kelly, A. M., Lindo, J. M., \& Packham, A. (2019). The Power of the IUD: Effects of Expanding Access to Contraception Through Title X Clinics. Obtenido de National Bureau of Economic Research: https://www.nber.org/papers/w25656

La Ferrara, E., Chong, A., \& Duryea, S. (Inter-American Development Bank Research Department). Soap Operas and Fertility: Evidence from Brazil. RES Working Papers 4573.

Nicolás, V., \& Mejía, F. (2015). La Inocencia Perdida: Un Contraste Regional de los Determinantes del Embarazo Adolescente en República Dominicana. Obtenido de Universidad Iberoamericana: http://empirica.do/download/la-inocencia-perdida-un-contraste-regional-de-los-determinantes-del-embarazo-adolescente-en-la-republica-dominicana? wpdmdl $=2175$

Observatorio de Políticas Sociales y Desarrollo. (2017). Embarazo y Adolescencia en República Dominicana: Un Estudio A Través de la Ecología Social. Santo Domingo: Observatorio de Políticas Sociales y Desarrollo.

Odimegwu, C., \& Mkwananzi, S. (2016). Factors Associated With Teen Pregnancy in sub-Saharian Africa: A Multi-Country Cross-Sectional Study. African Journal of Reproductive Health, 20(3), 94-107. 
Oettinger, G. S. (1999). The Effects of Sex Education on Teen Sexual Activity and Teen Pregnancy. Journal of Political Economy.

Organización de las Naciones Unidas, Departamento de Asuntos Económicos y Sociales, División de Población. (2017). World Population Prospects: The 2017 Revision. Nueva York: Naciones Unidas.

Paton, D. (2004). Random Behavior Or Rational Choice? Family Planning, Teenage Pregnancy and STI's. Royal Economic Society Annual Conference 2004, Royal Economic Society.

Pérez-Then, E., Miric, M., \& Vargas, T. (2011). El Embarazo en Adolescentes en República Dominicana: ¿Una Realidad en Transición? Santo Domingo: Profamilia.

Programa de Estudios Sociodemográficos (PESD). (2013). Costos del Embarazo y La Maternidad en la Adolescencia en la República Dominicana. Santo Domingo: Fondo de Población de las Naciones Unidas.

Programa de las Naciones Unidas para el Desarrollo República Dominicana (PNUD-RD). (2017). Embarazo Adolescente: Un Desafío Multidimensional Para Generar Opotunidades En El Ciclo de Vida. Santo Domingo: Amigo del Hogar.

Sabia. (2006). Does Sex Education Affect Sexual Behaviors and Health. Journal of Policy Analysis and Management, 25(4), 783-802. 